Results 8389 patients were included, 58\% were female, median age 68 years. Men and women appear to have a similar level of disease severity with no significant differences in the Respiratory Symptoms, Role Functioning and Health Perception domains of the QoL-B. There was however a significant difference in all other aspects of the QoL scores. Male patients had higher scores for Physical functioning, with $\beta$ coefficient 4.9 (95\% CI 3.3-6.5, p<0.0001) (indicating an average of 4.9 points higher for men when adjusted for confounding variables). Men also had higher scores for Emotional Functioning ( $\beta$ coefficient 3.6 (95\%CI 2.2-5.1), $p<0.0001$ ), Social Functioning ( $\beta$ coefficient 2.4 (95\% CI $0.7-4.1)$, $\mathrm{p}=0.007)$ and Vitality ( $\beta$ coefficient 2.5 (95\%CI $1.2-3.9$ ), $\mathrm{p}<0.0001)$. Across all domains, the elements with a large effect were Breathlessness, Comorbidities (with Depression and Anxiety commonly having a large impact), Pseudomonas aeruginosa infection and frequent exacerbations. Female patients nevertheless had greater disease impact even after adjusting for these variables.

Conclusions From this large dataset we show that despite similar levels of disease severity, women living with bronchiectasis are significantly more affected in physical, emotional and social functioning aspects of their lives than their male counterparts. This has implications for tailoring care and suggests a need for more holistic care for patients with bronchiectasis.

\section{S43 IN-HOSPITAL MORTALITY AND LENGTH OF STAY FOLLOWING CARDIOVASCULAR EVENTS AND INTERVENTIONS IN PEOPLE WITH BRONCHIECTASIS: A POPULATION BASED STUDY}

${ }^{1} \mathrm{M}$ Plowright, ${ }^{2} \mathrm{JP}$ Hutchinson, ${ }^{3} \mathrm{JK}$ Quint, ${ }^{2} \mathrm{RB}$ Hubbard, ${ }^{2} \mathrm{~V}$ Navaratnam. ${ }^{1}$ Nottingham University Medical School, University of Nottingham, Nottingham, UK; ${ }^{2}$ Division of Epidemiology and Public Health, University of Nottingham, Nottingham, UK; ${ }^{3}$ Department of Respiratory Epidemiology, Occupational Medicine and Public Health, National Heart and Lung Institute, Imperial College London, London, UK

\subsection{6/thoraxjnl-2017-210983.49}

Background The incidence and prevalence of bronchiectasis is increasing. Epidemiological studies have reported that people with bronchiectasis are at increased risk of cardiovascular comorbidities. However, there are limited data on outcomes after acute myocardial infarction (AMI), coronary artery bypass graft (CABG) or percutaneous angioplasty (PCI) amongst people with bronchiectasis. The aims of our study were to determine in-hospital mortality and mean length of hospital stay (LOHS) following AMI, CABG and PCI in people with and without bronchiectasis.

Methods We used data from the Nationwide Inpatient Sample, an anonymised stratified yearly sample of discharge records from community hospitals in the USA developed for the Healthcare Cost and Utilisation Project. People with a record of bronchiectasis from 2000 to 2011 were identified using the International Classification of Diseases (ICD-9-CM) codes. ICD-9-CM and procedure codes were also used to identify people with AMI, CABG and PCI. Multivariable logistic regression was used to estimate odds ratios for in-hospital mortality following AMI, CABG and PCI in people with and without bronchiectasis, adjusting for age and sex. We also compared mean LOHS following AMI, CABG and PCI between individuals with bronchiectasis and the general population.

Results We identified 101597 people with a record of bronchiectasis. The mean age of the cohort was 57.2 years (Standard Deviation 20.8) and 60.8\% were female. $2195(2.2 \%)$ individuals with bronchiectasis had an AMI, 366 (0.4\%) had undergone a CABG and $827(0.8 \%)$ underwent a PCI. In-hospital mortality amongst people with bronchiectasis following AMI, CABG and PCI was $12.5 \%, 3.6 \%$ and $2.9 \%$ respectively. After adjusting for age and sex, we found no difference in inhospital mortality following AMI, CABG or PCI in people with bronchiectasis compared to the general population (Table 1). Individuals with bronchiectasis had a longer mean LOHS following AMI, CABG and PCI (Table 1).

Conclusions Our findings suggest no difference in risk of death following AMI, CABG and PCI in people with bronchiectasis, which should be taken into account when counselling patients. However, individuals with bronchiectasis had a longer mean LOHS, which may impact healthcare resources and patient care pathways.

\section{S44 RCT EVALUATION OF THE BRONCHIECTASIS EMPOWERMENT TOOL SELF-MANAGEMENT INTERVENTION USING SELF-REPORTED QUESTIONNAIRES AND FOCUS GROUPS}

${ }^{1} \mathrm{C}$ Brockwell, ${ }^{1} \mathrm{AM}$ Wilson, ${ }^{1} \mathrm{~A}$ Clark, ${ }^{1} \mathrm{G}$ Barton, ${ }^{2} \mathrm{MC}$ Pasteur, ${ }^{3} \mathrm{R}$ Fleetcroft, ${ }^{4} \mathrm{JH}$ Hill, ${ }^{1}$ A Stöckl. 'School of Medicine and Health Sciences, University of East Anglia, Norwich, UK; ${ }^{2}$ Department of Respiratory Medicine, Norfolk and Norwich University Hospital NHS Foundation Trust, Norwich, UK; ${ }^{3}$ Acle Medical Practice, Bridewell Lane, Acle, UK; ${ }^{4}$ Norfolk Community Health and Care Trust, Norwich Community Hospital, Norwich, UK

10.1136/thoraxjnl-2017-210983.50

Abstract S43 Table 1 In-hospital mortality and mean length of hospital stay following AMI, CABG and PCI

\begin{tabular}{|c|c|c|c|c|c|c|c|}
\hline & $\begin{array}{l}\text { Number of people } \\
\text { with bronchiectasis } \\
(n=101,597)(\%)\end{array}$ & $\begin{array}{l}\text { No of people without } \\
\text { bronchiectasis } \\
(\mathrm{n}=77,666,681)(\%)\end{array}$ & $\begin{array}{l}\text { In-hospital mortality in } \\
\text { people with } \\
\text { bronchiectasis (\%) }\end{array}$ & $\begin{array}{l}\text { In-hospital mortality in } \\
\text { people without } \\
\text { bronchiectasis (\%) }\end{array}$ & $\begin{array}{l}\text { Adjusted } \\
\text { Odds Ratio } \\
\left(95 \% \mathrm{Cl}^{[i]}\right)^{*}\end{array}$ & $\begin{array}{l}\text { Mean LOHS } \\
\text { people with } \\
\text { bronchiectasis (SD) in } \\
\text { [iii] })\end{array}$ & $\begin{array}{l}\text { Mean LOHS (days) in } \\
\text { people without } \\
\text { bronchiectasis (SD) }\end{array}$ \\
\hline AMI & 2195 (2.16) & $2,423,961(3.12)$ & $12.5 \%$ & $10.1 \%$ & $\begin{array}{l}0.97(0.86- \\
1.10)\end{array}$ & $7.7(8.3)$ & $6.3(7.9)$ \\
\hline CABG & $366(0.36)$ & $732373(0.94)$ & $3.6 \%$ & $3.1 \%$ & $\begin{array}{l}0.90(0.51- \\
1.57)\end{array}$ & $11.4(8.7)$ & $9.8(8.5)$ \\
\hline $\mathrm{PCl}$ & $827(0.81)$ & $1,791,318(2.31)$ & $2.9 \%$ & $1.4 \%$ & $\begin{array}{l}1.40(0.93- \\
2.10)\end{array}$ & $6.0(8.1)$ & $3.2(4.2)$ \\
\hline
\end{tabular}

${ }^{*}$ Odds ratio adjusted for age and sex

'Confidence Interval

ii Length of Hospital Stay

iiistandard Deviation 
Introduction Evidenced self-management to guide patients in preserving their quality of life $(\mathrm{QoL})$ and moderate healthcare demands, is lacking in bronchiectasis though advocated in the guidelines. ${ }^{1}$ An expert patient plan has shown promise. $^{2}$

Aim To measure the impact on self-efficacy (confidence in dealing with disease), of the Bronchiectasis Empowerment Tool (BET) as part of a quality of life and economic evaluation.

Method 220 people from 6 UK hospitals were randomised (computerised) to standard treatment (including BTS physiotherapy leaflet and British Lung Foundation leaflet about bronchiectasis) alone or with the addition of BET. Participants had radiological diagnosis and at least one exacerbation within 12 months of enrolment. Individuals with cystic fibrosis, traction bronchiectasis and severe uncontrolled co-morbid disease were excluded. BET, (48 pages) comprising an action-plan based on the 2010 BTS Guidelines (1) and four educational sections: sputum, health changes, medications and health interactions (with notepads), was introduced using four brief telephone calls (totalling $24 \mathrm{~min}$ per person). All outcomes were selfreported: The primary outcome using the Self-Efficacy Measure for Chronic Disease (SEMCD) questionnaire at 12 months. QoL measures included St George's Respiratory Questionnaire, Lung Information Needs Questionnaire (LINQ) and non-validated questionnaires. Euroqol 5 Dimension (EQ5D) and healthcare utilisation questionnaires were used for economic evaluation. Participants received questionnaires quarterly for one year. Focus groups assessed acceptability.

Results 127 participants responded at 12 months. BET did not influence SEMCD (mean difference $(0.14 \quad(95 \%$ confidence interval $(95 \% \mathrm{CI}-0.37$ TO 0.64$), \mathrm{p}=0.59)$. NHS cost weren't significantly different between groups (mean difference $£ 335.94$, 95\% CI $£-444.97$ to $£ 1156.85$ ) nor were Quality adjusted life years derived from EQ5D data (mean difference $0.006,95 \% \mathrm{CI}-0.042$ to 0.053 ) or QoL. Focus group participants, diverse in severity, symptoms and isolation deemed the telephone element of BET acceptable.

Conclusion BET did not improve self-efficacy, QoL or diminish healthcare costs. Supporting literacy needs with increased contact (perhaps telephone) or novel methods of evaluation may reduce attrition in future self-management research using self-reported outcomes.

\section{S45 VALIDATION OF THE INCREMENTAL SHUTTLE WALK TEST AS A CLINICAL ENDPOINT IN BRONCHIECTASIS}

MK Cartlidge, AT Hill. University of Edinburgh, Edinburgh, UK

\subsection{6/thoraxjnl-2017-210983.51}

Introduction There is a need for objective clinical endpoints in bronchiectasis to evaluate response to new and existing therapies. Current clinical endpoints in use present their own challenges; 24 hour sputum volume is unreliable, microbial clearance is often only assessed qualitatively and there remains controversy over changes in microbial load. Quality of life questionnaires (St George's Respiratory Questionnaire (SGRQ), Leicester Cough Questionnaire, Bronchiectasis Health Questionnaire and Quality of Life-bronchiectasis are subjective outcome measures. The mean exacerbations and time to first exacerbation have been used in phase 3 trials but there remains debate regarding the definition of an exacerbation. The incremental shuttle walk test (ISWT) is a measure of functional exercise capacity and more objective than the $6 \mathrm{~min}$ walk test; an endpoint associated with survival in COPD.

Aim To evaluate the ISWT as an objective clinical endpoint in bronchiectasis by assessing its reliability, validity and responsiveness.

Methods To assess reliability 30 patients were invited 6 months apart whilst clinically well to perform the ISWT. To assess validity the ISWT scores were correlated with total SGRQ and activity scores in 94 patients (stable and exacerbation). To assess responsiveness 30 patients performed the ISWT pre-and post 14 days of intravenous antibiotic therapy for an exacerbation, 94 patients performed pre and post 14 days of oral antibiotics for exacerbation and sub-analysis from a previous study evaluated ISWT in 30 patients pre and post 12 months of nebulised gentamicin therapy.

Results There was no significant difference in median (IQR) distance walked at baseline $(390 \mathrm{~m}(225 \mathrm{~m}-462.5 \mathrm{~m}))$ and after 6 months $(400 \mathrm{~m}(260 \mathrm{~m}-480 \mathrm{~m}))$. There was a negative correlation between ISWT and total SGRQ $(r=-0.60)$ and the activity component $(\mathrm{r}=-0.64) \mathrm{p}<0.0001$. There was a median increase of $18.5 \%$ in distance walked with nebulised gentamicin, $16.3 \%$ with oral antibiotic therapy and $11.9 \%$

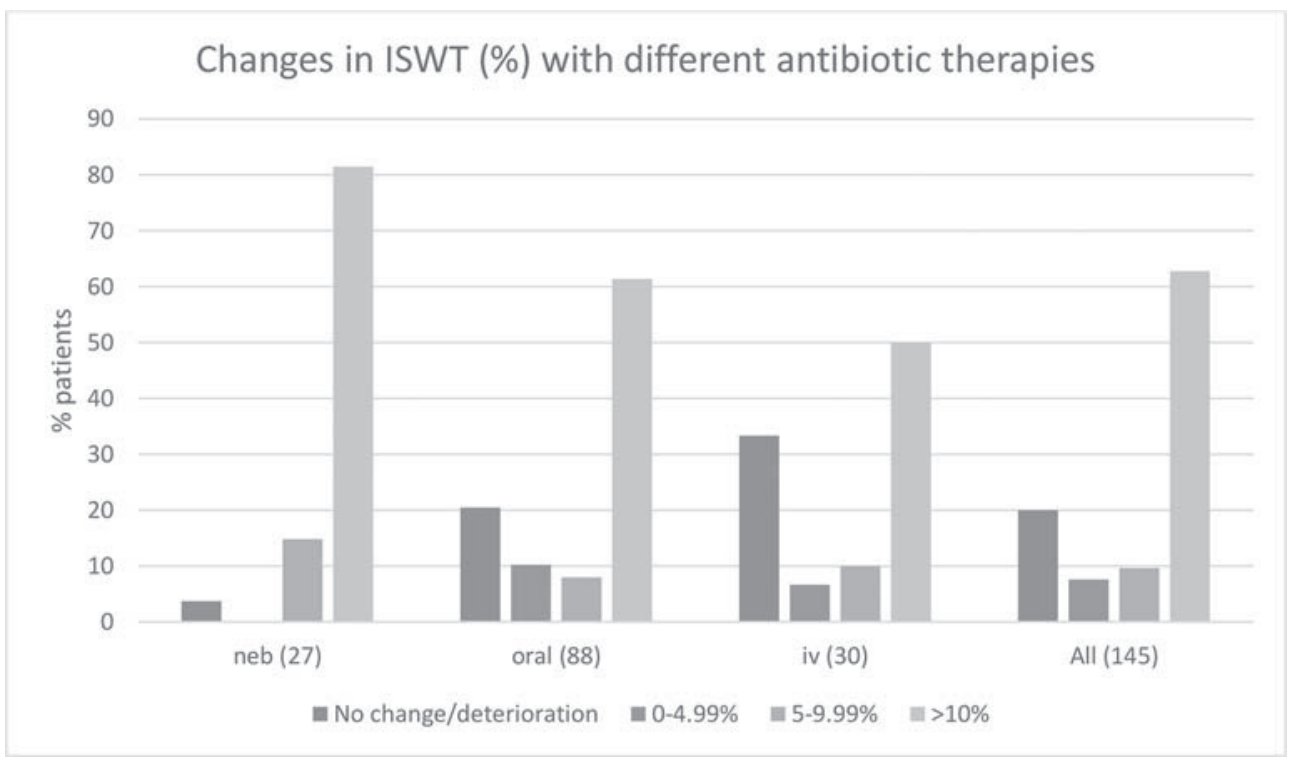

Abstract S45 Figure 1 Graph to show the changes (\%) in ISWT distance walked from baseline with different antibiotic therapies. 